\title{
LINEAR PRESERVERS ON STRICTLY UPPER TRIANGULAR MATRIX ALGEBRAS
}

\begin{abstract}
ALI A. JAFARIAN
Abstract. Let $\mathscr{S}_{n}(\mathbb{F})$ be the algebra of all $n \times n$ strictly upper triangular matrices over a filed $\mathbb{F}$. In this note, we characterize linear maps $\varphi: \mathscr{S}_{n}(\mathbb{F}) \rightarrow \mathscr{S}_{n}(\mathbb{F})$, with $|\mathbb{F}| \geqslant 3$, that preserve the adjugate function; i.e., $\operatorname{adj}(\varphi(A))=\varphi(\operatorname{adj}(A))$. Also, some results about rank-1 linear/additive preservers on $\mathscr{S}_{n}(\mathbb{F})$ and, more generally, on block strictly upper triangular algebras are obtained.
\end{abstract}

Mathematics subject classification (2010): 15A86, 15A15, 15A03.

Keywords and phrases: Linear adjugate preservers, linear ranks-1 preservers, additive rank-1 preservers, (block) strictly upper triangular matrices.

\section{REFERENCES}

[1] J. Bell, A. R. Sourour, Additive Rank-one Preserving Mappings on Matrix Algebras, Linear Algebra Appl. 312 (2000), 13-33.

[2] G. H. Chan, M. H. Lim, And K. K. Tan, Linear Preservers on Matrices, Linear Algebra Appl. 93 (1987), 67-80.

[3] W. L. Choor And M. H. Lim, Linear Preservers on Triangular Matrices, Linear Algebra Appl. 269 (1998), 241-255.

[4] G. Frobenius, Ü ber die Darstellung der endlichen Gruppen durch lineare Substitutionen, Sitzungsber, Deustch. Akad. Wiss. Berlin, 997-1015 (1897).

[5] R. Sinkhorn, Linear Adjugate Preservers on the Complex Matrices, Linear and Multilinear Algebra $12(1982 / 1983), 215-222$. 